University of Nebraska - Lincoln

DigitalCommons@University of Nebraska - Lincoln

$1-15-1999$

\title{
Evidence of dynamic Jahn-Teller distortions in two-dimensional crystalline molecular films
}

Jaewu Choi

University of Nebraska-Lincoln, jchoi@ece.eng.wayne.edu

Peter A. Dowben

University of Nebraska-Lincoln, pdowben@unl.edu

C.N. Borca

University of Nebraska-Lincoln

Shireen Adenwalla

University of Nebraska-Lincoln, sadenwalla1@unl.edu

A.V. Bune

University of Nebraska-Lincoln

See next page for additional authors

Follow this and additional works at: https://digitalcommons.unl.edu/physicsdowben

Part of the Physics Commons

Choi, Jaewu; Dowben, Peter A.; Borca, C.N.; Adenwalla, Shireen; Bune, A.V.; Ducharme, Stephen; Fridkin, V.M.; Palto, S.P.; and Petukhova, N., "Evidence of dynamic Jahn-Teller distortions in two-dimensional crystalline molecular films" (1999). Peter Dowben Publications. 36.

https://digitalcommons.unl.edu/physicsdowben/36

This Article is brought to you for free and open access by the Research Papers in Physics and Astronomy at DigitalCommons@University of Nebraska - Lincoln. It has been accepted for inclusion in Peter Dowben Publications by an authorized administrator of DigitalCommons@University of Nebraska - Lincoln. 


\section{Authors}

Jaewu Choi, Peter A. Dowben, C.N. Borca, Shireen Adenwalla, A.V. Bune, Stephen Ducharme, V.M. Fridkin, S.P. Palto, and N. Petukhova 


\title{
Evidence of dynamic Jahn-Teller distortions in two-dimensional crystalline molecular films
}

\author{
Jaewu Choi, P. A. Dowben,* C. N. Borca, Shireen Adenwalla, A. V. Bune, and Stephen Ducharme \\ Department of Physics and Astronomy, Behlen Laboratory of Physics, Center for Materials Research and Analysis, \\ University of Nebraska-Lincoln, Lincoln, Nebraska 68588-0111 \\ V. M. Fridkin, S. P. Palto, and N. Petukhova \\ Institute of Crystallography, The Russian Academy of Sciences, 117333 Moscow, Russia \\ (Received 27 February 1998; revised manuscript received 12 May 1998)
}

\begin{abstract}
The surface electronic structure in crystalline copolymer films of vinylidene fluoride $(70 \%)$ with trifluoroethylene $(30 \%)$ has been studied by photoemission and inverse photoemission as a function of alkali metal (sodium) doping. Sodium doping introduces at least two new states into the band (HOMO-LUMO) gap well away from the Fermi level. While the sodium-doped copolymer is observed to resemble an $n$-type semiconductor, the change in electronic structure with temperature suggests that dynamic distortions lead to a photoemission initial state splitting of the lower Hubbard-like bands. There is a decrease in the effective Debye temperature with sodium doping which may, in turn, enhance the dynamic Jahn-Teller distortion.

[S0163-1829(99)06603-5]
\end{abstract}

\section{INTRODUCTION}

There exist a large number of studies of the alkali doping of large organic molecular overlayers. Upon doping, new states should appear at the chemical potential (the Fermi level) in the absence of a correlation energy, as in the case of alkali doped poly-hexyl-thiophene. ${ }^{1}$ In fact there are many examples where alkali doping creates states below the Fermi level (the occupied side), but within the host material (the undoped material) band gap, i.e., between the highest occupied molecular orbital (HOMO) and the lowest unoccupied molecular orbital (LUMO) of the undoped system. The appearance of states in the HOMO-LUMO gap has been reported for alkali doping of poly(para-phenylenevinylene), ${ }^{2}$ $\alpha, \omega$-diphyltetradecaheptaene, ${ }^{3,4}$ poly(pyridine-2,5-diyl), ${ }^{5}$ poly $\left(2,2^{\prime}\right.$-bipyridine-5,5'-diyl) (Ref. 5) and 1,2dicarbadodecaborane. ${ }^{6}$ These alkali induced states have been variously ascribed to the formation of lower Hubbard bands, ${ }^{6}$ bipolaron states, ${ }^{5,2}$ and a soliton-antisoliton pair. ${ }^{3,4}$

The observation of two photoemission states, in place of a single molecular orbital, can be a consequence of either the photoemission initial state or final state (or indeed have contributions from both). Such multiplication of the expected number of photoemission features is a known result of symmetry breaking in the photoemission process ${ }^{7}$ and other final state effects. Initial state effects such as a soliton-antisoliton pairs or bipolaron formation ${ }^{2-5}$ have also been invoked, but such initial-state effects, as well as final-state effects, are generally unlikely to exhibit substantial temperature dependence and certainly these effects are unlikely to become more pronounced in photoemission with increased temperature (quite the opposite is generally expected). For solitonantisoliton pairs, static Jahn-Teller distortions, bipolaron formation or symmetry breaking in the spectroscopy final state, increasing temperature should lead to a decrease in the ability to resolve the two photoemission states as with increasing temperature the polarons should unbind and broadening effects due to dynamic motion should play an increasing role.
An increase in the ability to resolve the two photoemission states with increasing temperature would support the models of dynamic Jahn-Teller distortion in molecular systems such as those proposed by Santoro and co-workers. ${ }^{8}$

Recently, a new ferroelectric phase transition was observed at $20{ }^{\circ} \mathrm{C}$, distinct from the bulk transition ferroelectric transition at $80{ }^{\circ} \mathrm{C}$, in two dimensional films of ferroelectric vinylidene-fluoride polymers. ${ }^{9}$ This new transition was demonstrated to be a surface ferroelectric transition accompanied by a change in the surface electronic structure, using a combination of photoemission and inverse photoemission measurements. ${ }^{10}$ In this work we characterize the influence of cumulative alkali doping on the change in surface metallicity and electronic structure, as a function of temperature in crystalline 5-ML films (5 ML) of $\mathrm{P}(\mathrm{VDF}-\mathrm{TrFE}$ 70:30), a random copolymer of vinylidene fluoride with trifluoroethylene, consisting of all trans - $\left[\left(-\mathrm{CH}_{2}-\mathrm{CF}_{2}\right)_{0.70^{-}}\right.$ $\left.\left(\mathrm{CHF}-\mathrm{CF}_{2}-\right)_{0.30}\right]_{n}$ - chains. $^{9,11-12}$

\section{EXPERIMENT}

The films were formed by Langmuir-Blodgett monolayer deposition from a water subphase as described elsewhere. ${ }^{13}$ The films were deposited on silicon for photoemission studies and on an aluminum-coated glass substrate with a sputtered aluminum top electrode for dielectric studies. The inverse photoemission (IPES) and photoemission (PES) studies were performed in a U.H.V. chamber described in detail elsewhere ${ }^{14}$ with a base pressure better than 1 $\times 10^{-10}$ Torr. The films were prepared by gentle annealing to $200{ }^{\circ} \mathrm{C}$ in vacuo and surface composition characterized with core-level spectroscopy (XPS). The valence-band photoemission spectra were acquired with a helium lamp operated at the He I emission line $(21.2 \mathrm{eV})$ and a Physical Electronics model 365A hemispherical analyzer. The combined resolution was $50 \mathrm{meV}$, as determined from the Fermi edge of gold. The photoemission spectra shown in this work were taken with $60^{\circ}$ incidence angle and normal emission. 


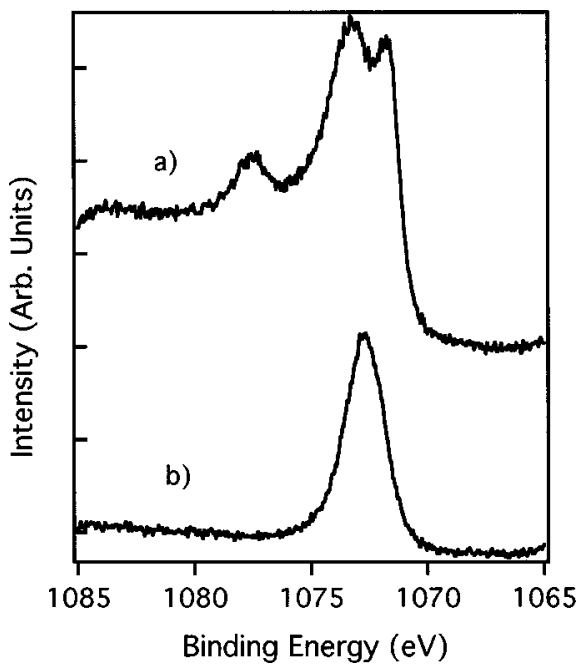

FIG. 1. The sodium $1 s$ core level after (a) deposition of $1 \mathrm{ML}$ equivalent of sodium on vinylidene fluoride $(70 \%)$ with trifluoroethylene (30\%) and (b) after annealing to $423 \mathrm{~K}$.

For the IPES studies, a Geiger-Müller detector with a $\mathrm{CaF}_{2}$ window was used in conjunction with an Erdman-Zipf electron gun. ${ }^{15}$ The overall energy resolution was $\sim 400$ $\mathrm{meV}$. All IPES spectra were collected with the electrons incident normal to the surface. The Fermi level for both inverse photoemission and photoemission was established using a gold reference and the temperature was determined using a chromel-alumel thermocouple. A SAES Na getter was used to dope the $\mathrm{P}(\mathrm{VDF}-\mathrm{TrFE})$ film. The sodium coverage was determined using a quartz crystal thin film monitor calibrated by XPS. The films were annealed to ensure a uniform distribution of alkali metal throughout the film. XPS measurements were taken using magnesium $K_{\alpha}$ radiation $(1253.6 \mathrm{eV})$ and the binding energies are with respect to the Fermi level of the gold reference.

\section{ALKALI DOPING}

The sodium, as deposited on a 5-ML film of P(VDF-TrFE 70:30) (70\% vinylidene with $30 \%$ trifluoroethylene) at 200 $\mathrm{K}$, exhibits a $\mathrm{Na} 1 s$ line shape with two distinct features characteristic of an inhomogeneous distribution of alkali metal, as seen in Fig. 1. In addition, there is a clear plasmonlike loss feature at $1077 \mathrm{eV}$ binding energy characteristic of sodium clustering. ${ }^{16}$ Annealing the film following sodium deposition leads to a far more uniform distribution of alkali metal without any evidence of metallic cluster formation. As seen in Fig. 1, upon annealing to $350 \mathrm{~K}$ (or above), the $\mathrm{Na} 1 s$ core level exhibits only a single feature and suppression of the plasmonlike loss. This is characteristic of a uniform distribution of alkali metal at roughly equivalent binding sites.

In Fig. 2 we present a series of PES and IPES spectra of an undoped 5-ML film of P(VDF-TrFE 70:30). The inverse photoemission places the Fermi level (the chemical potential) near the unoccupied conduction band, while little or no density of states is observed near the Fermi level in photoemission, characteristic of an $n$-type semiconductor as noted elsewhere. ${ }^{10}$ With a film only 5 ML thick, charging is not likely to be a dominant effect and we found no evidence for surface photovoltage effects.

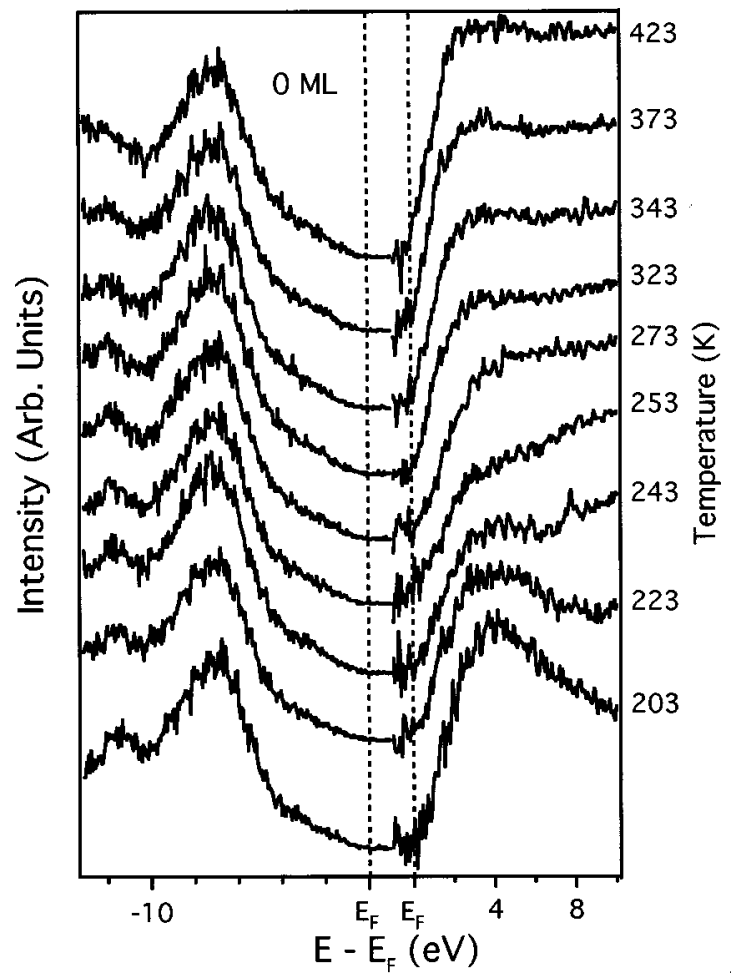

FIG. 2. Normal incidence inverse photoemission spectra (right) and normal emission photoemission spectra (left) of a 5-ML crystalline copolymer film of vinylidene fluoride (70\%) with trifluoroethylene $(30 \%)$. The spectra were taken as a function of temperature. Little difference was observed between spectra taken with increasing temperature as opposed to decreasing temperature (no hysteresis).

Photoemission and inverse photoemission spectra have been taken following deposition of 1-, 2-, and 4-ML equivalents of sodium and annealing to $423 \mathrm{~K}$ with the most heavily doped samples shown in Fig. 3. Difference spectra that subtract out the contributions of the undoped P(VDFTrFE 70:30) indicate that alkali metal doping introduces an occupied density of states in the valence-band region at about $5 \mathrm{eV}$ binding energy with respect to the common Fermi level, as seen in Figs. 4(a)-4(d). With increasing temperature and large doping levels, this sodium-induced density of states can be resolved into two distinct features at 3.5 and $5.5 \mathrm{eV}$ binding energy with respect to $E_{F}$. This is similar to the sodium-induced states in orthocarborane $(1,2-$ dicarbadodecaborane) at 2.8 and $4.3 \mathrm{eV}$ binding energy with respect to the Fermi level ${ }^{6}$ but is at far greater binding energy (with respect to the Fermi level) than was observed with alkali-metal doping of poly(para-phenylenevinylene) at 0.9 and $2.8 \mathrm{eV},{ }^{2} \alpha, \omega$-diphyltetradecaheptaene at 0.9 and 1.7 $\mathrm{eV},{ }^{3,4}$ poly(pyridine-2,5-diyl) at 0.9 and $3.1 \mathrm{eV}^{5}$ and poly (2,2'-bipyridine-5,5'-diyl) at 0.9 and $3.3 \mathrm{eV} .^{5}$ All of these large molecular systems exhibit, in common, a large HOMO-LUMO gap. The similarities, in the influence of alkali-metal doping for various systems, are indicated in Fig. 4.

The fact that alkali doping introduces similar changes in all of these molecular systems suggests that similar mechanisms are involved and that the correct model should be insensitive to the molecular structure. Yet the models used to 


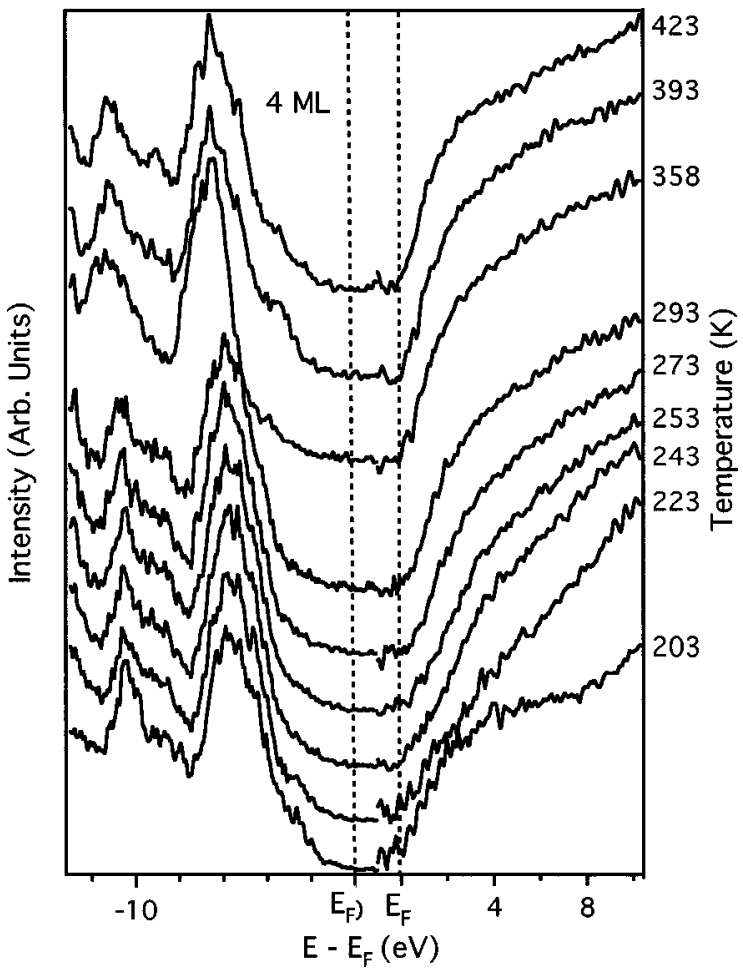

FIG. 3. Normal incidence inverse photoemission spectra (right) and normal emission photoemission spectra (left) of a 5-ML crystalline copolymer film of vinylidene fluoride $(70 \%)$ with trifluoroethylene (30\%) doped with $4 \mathrm{ML}$ equivalent of sodium. The spectra were taken as a function of temperature.

explain these alkali induced states are diverse. We have already noted that the alkali-metal states have been attributed to lower Hubbard bands, ${ }^{6}$ bipolaron states, ${ }^{2,5}$ or a solitonantisoliton pair. ${ }^{3,4}$ While the models are not necessarily mutually exclusive, a model cannot be generally applicable unless it applies in all of the above molecular host systems (or at least most). Insight into the most applicable model can be, in part, gained through an inspection of the temperature dependence of the sodium induced states in the HOMO-LUMO gap, as indicated in the Introduction. A definitive comparison among all of these systems requires combined photoemission and inverse photoemission data so that the relative positions of the chemical potential (the Fermi level relative to the conduction and valence bands) can be ascertained in each. Corelevel spectroscopy demonstrating a uniform distribution of alkali metal is also essential.

\section{EVIDENCE FOR STRONG ELECTRON-PHONON COUPLING}

From the valence-band features shown in the photoemission spectra in Figs. 2-5, in particular, the molecular orbital at $12 \mathrm{eV}$ binding energy with respect to the Fermi level (possibly related to the C-F bond ${ }^{10}$ ), we can extract an effective Debye temperature with increasing sodium doping. The effective Debye temperatures can be deduced from the change of the photoemission intensity as a function of temperature. ${ }^{17,18}$ The vertical dynamic motion of the surface atoms influences intensity of the photoemission features, which exponentially decays with Debye-Waller factor $W:^{18}$

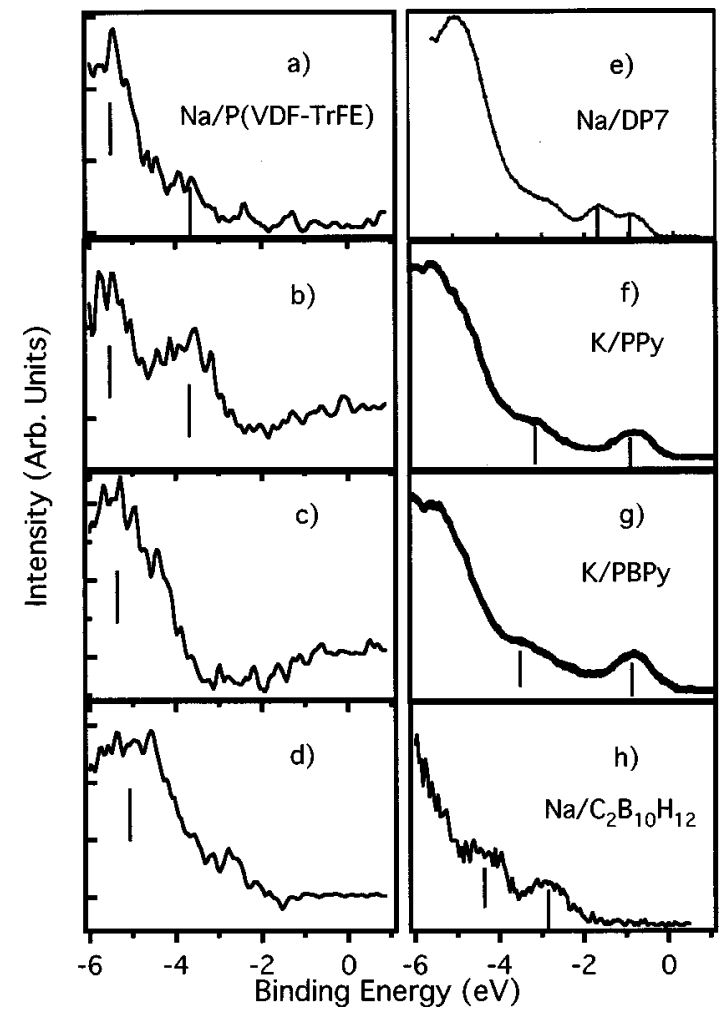

FIG. 4. The valence-band difference spectra of the $4 \mathrm{ML}$ sodium doped $\mathrm{P}(\mathrm{VDF}-\mathrm{TrFE})$ film with respect to the undoped valence-band spectra (obtained by subtracting the PES spectra without alkalimetal doping from those spectra with alkali-metal doping) at temperatures of (a) $423 \mathrm{~K}$, (b) $393 \mathrm{~K}$, (c) $293 \mathrm{~K}$, and (d) $203 \mathrm{~K}$, respectively. The corresponding valence-band spectra are shown for (e) sodium doped DP7( $\alpha, \omega$-diphenyltetradecaheptaene) taken from Refs. 3, 4, (f) potassium doped on the PPy[poly(pyridine-2,5-diyl)] taken from Ref. 5, (g) potassium doped on the PBPy[poly(2,2'-bipyridine-5,5'-diyl) taken from Ref. 5, and (h) sodium doped on the orthocarborane (closo-1.2-dicarbadodecaborane) taken from Ref. 6.

$$
I=I_{0} \exp (-2 W)
$$

where $W$ is proportional to the mean square displacement of the atoms due to the thermal vibration. This vertical displacement is a function of the effective Debye temperature $\theta_{D}$ and the sample temperature $T$ as ${ }^{19}$

$$
\left\langle\Delta r^{2}\right\rangle=\frac{3 h^{2} T}{4 \pi^{2} M k_{B} \theta_{D}^{2}},
$$

where $M$ is the mass of the scattering center, in this study, the carbon that forms the polymer chain is chosen as a scattering center. The Debye-Waller factor $W$ is a function of the momentum transfer $\Delta k$ and the mean-square displacement of the atoms according to

$$
2 W=|\Delta k|^{2}\left\langle\Delta r^{2}\right\rangle .
$$

As shown in Figs. 2, and 5, the intensity of the features at around the binding energy $12 \mathrm{eV}$ (for $0 \mathrm{ML} \mathrm{Na}$ doping), and $11 \mathrm{eV}$ (with higher levels of Na doping) decreased with increasing of the sample temperature. The dependence of the intensity on temperature is shown in Fig. 5. The effective Debye temperatures for each sodium coverage was fit to the 


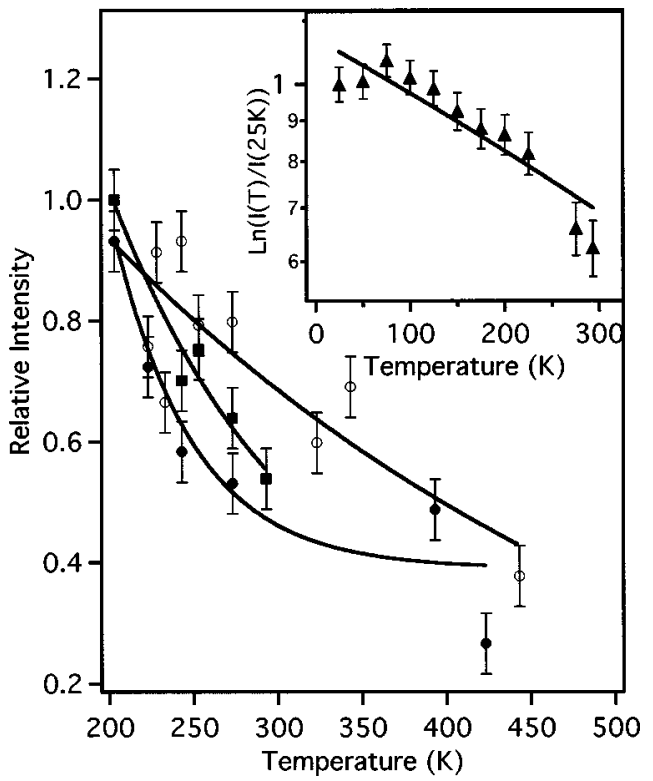

FIG. 5. The relative intensities of the photoemission features at $12 \mathrm{eV}$ (0 ML Na doping), and $11 \mathrm{eV}$ (2 and $4 \mathrm{ML}$ Na doping) with respect to the intensity at $203 \mathrm{~K}$ as a function of temperature. The solid lines correspond to effective Debye temperatures of $(\bigcirc) 53 \mathrm{~K}$ (0 ML Na), (-) $21 \mathrm{~K}(2 \mathrm{ML} \mathrm{Na})$, and (ם) $29 \mathrm{~K}$ (4 ML Na). The inset is the effective bulk Debye temperature of $69 \mathrm{~K}$ fitted to data from neutron scattering for an undoped film nominally $50 \mathrm{ML}$ thick (Ref. 20).

Debye formalism [Eq. (1)] yielding approximately $50 \mathrm{~K}$ for 0 ML Na doping, 20-30 K with Na doping, representative of dynamic motion normal to the surface.

Confirmation that the temperature dependence of this photoemission feature is indeed representative of the effective bulk Debye temperature, from motion normal to the film, comes from comparison with neutron scattering measurements ${ }^{20}$ shown in the insert to Fig. 5. An effective Debye temperature of $69 \pm 3.5 \mathrm{~K}$, obtained from neutron scattering, compares well with an effective Debye temperature of $53 \pm 11 \mathrm{~K}$ obtained from photoemission for the undoped P(VDF-TrFE 70:30) film. The differences have been attributed to greater surface sensitivity in photoemission as well as possible systematic errors from the analysis (not the data) but in any case, this value is much higher than the anticipated effective surface Debye temperature of approximately $26 \mathrm{~K}$ based on inverse photoemission. ${ }^{10}$

There is a decrease in this effective bulk-like Debye temperature with sodium doping. This may also explain the changes in the binding energy of the lowest unoccupied molecular orbitals with temperature. In lattices dominated by dynamic Jahn-Teller distortions, cooperative Jahn-Teller distortions are to be expected-driven by the intermolecular elastic coupling. ${ }^{8}$ Such cooperative distortions could be static and become manifest in the molecular crystal as a conformational change ${ }^{21}$ in the molecular P(VDF-TrFE 70:30) chain. The all-trans configuration of P(VDF-TrFE 70:30) (Refs. 11, 21) might be enhanced or suppressed at low temperatures. Steric and electrostatic intramolecular interactions ${ }^{11,21}$ might be overcome by cooperative Jahn-Teller distortions if the effective Debye temperature of the chain conformation is sufficiently small.
Such an influence of the phonon modes on the electronic structure is not entirely unexpected and has been, to some extent, anticipated by theory. ${ }^{8}$ Not all aspects of sodium doping are consistent with expectations. We would anticipate that the dielectric response would increase with sodium doping. This should lead to a damping of the cooperative phonon modes and an increase of the effective Debye temperature. While there are some indications that the Debye temperature may rise with very high sodium doping levels, this is not clearly observed nor are the results at this stage definitive. The sodium doping may also lead to the loss or diminution of the possible conformational change could be the origin of the shift in the lowest unoccupied molecular orbital binding energy below $20^{\circ} \mathrm{C}$.

Dynamic or static, increased Jahn-Teller distortions could split the lower Hubbard band, similar to the alkali-metalinduced states, into more than one feature. Since the observed splitting of the alkali-metal-induced states occurs with increasing temperature we cannot attribute this effect to a Jahn-Teller distortion or symmetry breaking introduced by the photoemission process. ${ }^{7}$ The change in the alkali-metalinduced features with increasing temperature result in two states each with a smaller width than the single valence-band feature observed at lower temperatures (Fig. 4). This cannot be explained by the Debye scattering due to the limited resolution of the experiment, though the diminution of these photoemission features with increasing temperature is consistent with a low effective Debye temperature.

Symmetry-lowering ferroelectric phase transitions are usually attributed to Jahn-Teller or pseudo-Jahn-Teller distortions where doping tends to lower the transition temperature by weakening the Jahn-Teller interaction. ${ }^{22,23}$ Ferroelectricity in PVDF and its copolymers has been modeled statistically based on steric interactions, strong intrachain dihedral forces coupled with a mean field of relatively weak interchain van der Waals forces. ${ }^{24}$ The present results suggest that these models should be examined for the appearance of intrachain soft modes near the transition temperature.

Whatever model is applied to alkali doping of molecular systems, we must account for a strong temperature dependence. This suggests that electron-phonon coupling plays an important role in these systems. We postulate the existence of Jahn-Teller distortions in these systems at the higher temperatures. This has important implications for the interpretation of the ferroelectric-paraelectric phase transition exhibited by the $\mathrm{P}(\mathrm{VDF}-\mathrm{TrFE})$ system. ${ }^{9-13}$

\section{OTHER TEMPERATURE-DEPENDENT EFFECTS}

The influence of temperature on the electronic structure in the 5-ML $\mathrm{P}(\mathrm{VDF}-\mathrm{TrFE} 70: 30)$ film is quite pronounced in IPES, as seen in Fig. 2. With increased temperature, the unoccupied molecular orbitals of $\mathrm{P}(\mathrm{VDF}-\mathrm{TrFE})$ shift to lower binding energies, driving the conduction band into the Fermi level. Substantive changes in the unoccupied density of states are observed as a function of temperature in the vicinity of the Fermi level, as summarized in Fig. 6(a). This result, taken in conjunction with the shift of the unoccupied density of states toward the Fermi level, indicates that the insulator gap is closing with increasing temperature. ${ }^{10}$

The increase in the observed density of states at the Fermi 


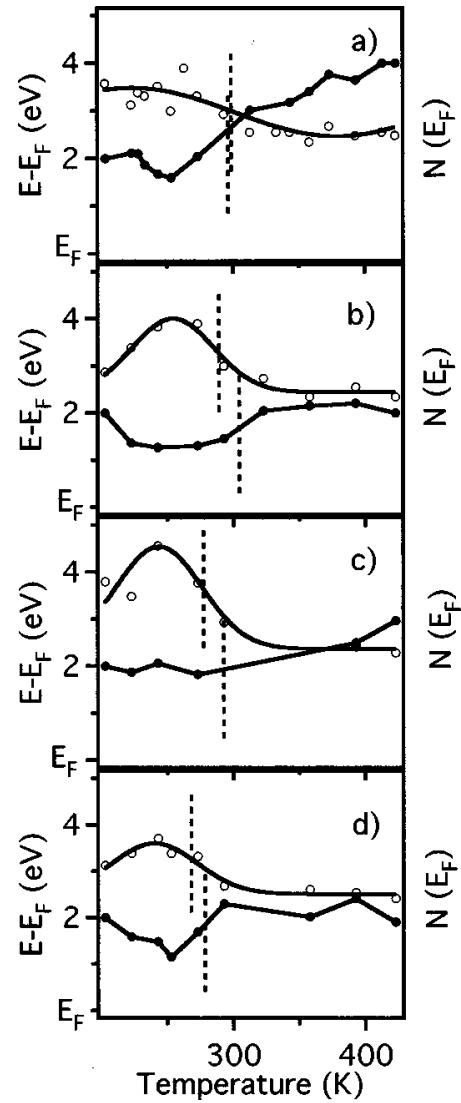

FIG. 6. The binding energy shift $(\bigcirc)$ of the LUMO states and the summation of the occupied and unoccupied density of states near the Fermi level (O) for 0 ML (a), 1 ML (b), 2 ML (c), and 4 ML (d) sodium doping.

level and a shift in binding energy of the LUMO states toward the Fermi level [Fig. 6(a)] in the crystalline ferroelectric polymer coincides with the ferroelectric transition observed at $20^{\circ} \mathrm{C}$ (on heating), ${ }^{9,10}$ The surface ferroelectric phase $\left(T<20^{\circ} \mathrm{C}\right)$ is characteristically insulating with a clearly established band gap. Since we observe no increase in the occupied density of states (from PES) for the undoped films with increasing temperature, we cannot conclusively say that the corresponding surface paraelectric phase $(T$ $>20{ }^{\circ} \mathrm{C}$ ) is metallic but given the changes in conductivity and electronic structure, it certainly resembles a highly doped semiconductor (semimetal). ${ }^{10}$ Thus this ferroelectric phase transition at about $20^{\circ} \mathrm{C}$ is accompanied by a change in electronic structure that closely resembles a nonmetal-tometal transition in many respects.

A change in the unoccupied density of states near the Fermi level is also observed with Na doping of the film. The dramatic increase in the density of states at the Fermi level occurs at increasingly lower temperatures with increasing sodium doping levels (Fig. 6). With increasing sodium doping, there is a pronounced shift away from the Fermi level then back toward the Fermi level with increasing temperature (Figs. 4 and 6). These effects are least pronounced at the highest doping levels. While these changes may be associated with a coupling of the surface ferroelectric to paraelectric transition with a semiconductor-to-semimetal-like transition, as was shown with the undoped films, ${ }^{10}$ the changes in conductivity as well as the surface ferroelectric transition in the sodium doped $\mathrm{P}(\mathrm{VDF}-\mathrm{TrFE})$ crystalline polymer films have not been characterized. Nonetheless, the dramatic increase in the unoccupied density of states right at the Fermi energy, observed in inverse photoemission, is persistently at approximately $0-20{ }^{\circ} \mathrm{C}$ independent of the sodium doping level.

\section{SUMMARY}

We find evidence in support of strong electron-phonon coupling in two-dimensional films of the copolymer of vinylidene fluoride (70\%) with trifluoroethylene $(30 \%)$. This is evident in the correlation between the measured effective Debye temperature and the extent of the binding energy shifts in the lowest unoccupied molecular orbitals with temperature. The splitting of the occupied gap states, which were introduced by sodium doping, with increasing temperature suggests that at least some of the electron-phonon coupling is associated with dynamic Jahn-Teller distortions. Such splittings of states induced by sodium doping may have been incorrectly interpreted in some experimental systems in the past and similar temperature-dependent studies of these systems are indicated.

The electron-phonon coupling may be manifest in configurational changes in the polymeric chain that occur as cooperative distortions but structural studies are certainly needed at this stage if these issues are to be further clarified. While static Jahn-Teller distortions, bipolarons, or antisolition-soliton pairs cannot be eliminated based upon our data, the strong temperature dependence of the photoemission and the low Debye temperatures implicate dynamic motion as a major contribution to the experimental electronic structure.

\section{ACKNOWLEDGMENTS}

This work was supported by the National Science Foundation, the Office of Naval Research, and the Nebraska Research Initiative through the Center for Materials Research and Analysis. S.P.P. and N.P. were supported by INTAS Project No. N93-1700.
*Author to whom correspondence should be addressed. FAX: 402472-2879. Electronic address: pdowben@unl.edu

${ }^{1}$ M. Lögdlund, R. Lazzaroni, S. Stafström, W. R. Salaneck, and J. L. Brédas, Phys. Rev. Lett. 63, 1841 (1989).

${ }^{2}$ M. Fahlman, D. Beljonne, M. Lögdlund, R. H. Friend, A. B. Holmes, J. L. Brédas, and W. R. Salaneck, Chem. Phys. Lett. 214, 327 (1993).

${ }^{3}$ M. Lögdlund, P. Dannetun, S. Stafström, W. R. Salaneck, M. G.
Ramsey, C. W. Spangler, C. Fredricksson, and J. L. Brédas, Phys. Rev. Lett. 70, 970 (1993).

${ }^{4}$ P. Dannetun, M. Lögdlund, C. Fredriksson, R. Lazzaroni, C. Fauquet, S. Stafström, C. W. Spangler, J. L. Brédas, and W. R. Salaneck, J. Chem. Phys. 100, 6765 (1994).

${ }^{5}$ T. Miyamae, D. Yoshimura, H. Ishii, Y. Ouchi, T. Miyazaki, T. Koike, T. Yamamoto, and K. Seki, J. Electron Spectrosc. Relat. Phenom. 78, 399 (1996). 
${ }^{6}$ D. N. McIlroy, C. Waldfried, T. McAvoy, J.-W. Choi, P. Dowben, and D. Heskett, Chem. Phys. Lett. 22, 1048 (1997).

${ }^{7}$ J. Eiding, W. Domcke, W. Huber, and H.-P. Steinrück, Chem. Phys. Lett. 180, 133 (1991).

${ }^{8}$ G. Santoro, L. Guidoni, A. Parola, and E. Tosatti, Phys. Rev. B 55, 16168 (1997).

${ }^{9}$ A. V. Bune, V. M. Fridkin, S. Ducharme, L. M. Blinov, S. P. Palto, A. Sorokin, S. G. Yudin, and A. Zlatkin, Nature (London) 391, 874 (1998).

${ }^{10}$ Jaewu Choi, P. A. Dowben, Shawn Pebley, A. Bune, Stephen Ducharme, V. M. Fridkin, S. P. Palto, and N. Petukhova, Phys. Rev. Lett. 80, 1328 (1998).

${ }^{11}$ A. Lovinger, Science 220, 1115 (1983).

${ }^{12}$ S. Ducharme, A. Bune, V. M. Fridkin, L. Blinov, S. Palto, N. Petukhova, and S. Yudin, Phys. Rev. B 57, 25 (1998).

${ }^{13}$ S. Palto et al., Ferroelectr. Lett. Sect. 19, 65 (1995); L. M. Blinov, Sov. Phys. Usp. 31, 623 (1988).

${ }^{14}$ D. N. McIlroy et al., Phys. Rev. B 54, 17438 (1996).
${ }^{15}$ P. W. Erdman and E. C. Zipf, Rev. Sci. Instrum. 53, 225 (1982).

${ }^{16}$ T. Reiners, C. Ellert, M. Schmidt, and H. Haberland, Phys. Rev. Lett. 74, 1558 (1995)

${ }^{17}$ C. Waldfried, D. N. McIlroy, Jiandi Zhang, P. A. Dowben, G. A. Katrich, and E. W. Plummer, Surf. Sci. 363, 296 (1996).

${ }^{18}$ N. J. Shevchik, Phys. Rev. B 16, 3428 (1977) and references therein.

${ }^{19}$ L. J. Clarke, Surface Crystallography-An Introduction to Low Energy Electron Diffraction (Wiley, New York, 1985).

${ }^{20}$ C. N. Borca, Jaewu Choi, Shireen Adenwalla, Stephen Ducharme, P. A. Dowben, Lee Robertson, V. M. Fridkin, S. P. Palto, and N. Petukhova, Appl. Phys. Lett. (to be published).

${ }^{21}$ Takeo Furukawa, Phase Transit. 18, 143 (1989).

${ }^{22}$ N. N. Kristofel' and P. I. Konsin, Sov. Phys. Solid State 13, 2969 (1972).

${ }^{23}$ G. Chanussot, Ferroelectrics 8, 671 (1974).

${ }^{24}$ R. Zhang and P. L. Taylor, J. Appl. Phys. 73, 1395 (1993). 